\title{
Responsabilidad penal del farmacéutico en el despacho de medicamentos
}

Pharmacist's criminal liability on dispensing medicines

\section{Freddy Arias}

Farmacéutico, Licenciado en Farmacia. Universidad de Costa Rica, San José, Costa Rica.

\section{Vivian Luna}

Farmacéutica, Licenciada en Farmacia. Colegio de Farmacéuticos de Costa Rica, San José, Costa Rica.

Resumen: La doctrina penal no ha profundizado en el análisis de la responsabilidad del profesional en farmacia en el despacho de medicamentos, el cual es un acto complejo en el que participan varios profesionales del equipo de salud. Normalmente estos los integrantes de estos equipos parten de la confianza en el correcto actuar de los miembros del equipo, sin embargo el profesional en farmacia parte de la desconfianza en las actuaciones de otros profesionales, por lo cual resulta de interés analizar su actuación en este acto profesional en particular.

Palabras claves: Responsabilidad penal del profesional farmacéutico; Farmacia, lex artis.

Resumo: A doutrina penal não avançou muito na análise da responsabilidade do farmacêutico em relação à dispensação de medicamentos, um ato complexo do qual participam vários profissionais da equipe de saúde. Normalmente, os integrantes dessas equipes agem em confiança na atuação dos seus demais membros, enquanto a atuação do farmacêutico parte da desconfiança na atuação dos demais profissionais. Em vista disso, é interessante analisar sua atuação nesse ato profissional em particular.

Palavras chaves: Responsabilidade penal do farmacêutico; Farmácia, lex artis.

\begin{abstract}
The penal doctrine has not performed an in depth analysis of the pharmaceutical professionals responsibility in the delivery of medication which is a complex act that requires the participation of several health professionals. Typically the health care professionals inherently trust in the actions of their colleagues however, the pharmaceutical professionals distrust the actions of other professionals, resulting in an interest to analyze their behavior in regards to this professional act.
\end{abstract}

Key-words: Criminal liability; Pharmacy, lex artis. 


\section{Antecedentes}

Los profesionales de la salud se dedican al tratamiento de enfermedades que, la mayoría de las veces, requiere una evaluación de riesgos para el paciente. El profesional requiere conocimiento y experticia, que permita a los pacientes hacer frente a la incertidumbre de su evolución (Horwitz, 2006). En la relación entre el profesional sanitario y el paciente, es de vital importancia la confianza entre ambas partes $y$, en especial, se requiere que el profesional sea digno de esta confianza, debido a que el paciente se encuentra en una situación desventajosa porque no se encuentran en igualdad de condiciones; el paciente no posee los mismos recursos y elementos técnicos para tomar las decisiones para su tratamiento, y debe confiar en que el profesional actuará de la mejor manera de acuerdo a su criterio profesional.

Actualmente, la relación entre los profesionales en salud y la sociedad se construye en un ambiente de confianza y duda. La confianza en el profesional debe ser desarrollada considerando situaciones de incertidumbre crecientes en los últimos años, propias del ejercicio del profesional en el campo de la salud; desarrollo de la tecnología en ciencias médicas, aumento en la especialización y complejidad de los diagnósticos y los tratamientos; mayor acceso a la información por parte del paciente y mayor conciencia de sus derechos, lo que ha disminuido la confianza del paciente en el profesional y ha causado un aumento en el número de reclamos de responsabilidad al profesional en salud (Portero, 2001).

El farmacéutico es el profesional en salud responsable de realizar el despacho de recetas de medicamentos consecuencia de una valoración, diagnóstico del profesional en medicina. El farmacéutico debe corroborar que el medicamento prescrito sea el idóneo y está en la obligación de rechazar el despacho de las recetas que no estén conforme a las exigencias científicas, legales y reglamentarias. Este control cruzado tiene su motivación en garantizar el resguardo de la salud del paciente al prevenir errores de prescripción. El inadecuado despacho de medicamentos podría generar responsabilidad penal si se genera un resultado dañoso para algún paciente.

En el examen de la responsabilidad penal por un delito culposo por mal despacho de medicamentos, debe considerarse de manera individual la violación al deber de cuidado como desviación de la lex artis farmacéutica. En el caso de equipos de trabajo interdisciplinarios, el deber de cuidado se complementa por el principio de confianza que se analiza según el tipo de división del trabajo, horizontal o vertical. 
Según Sánchez-Caro y Abellán, los casos típicos de responsabilidad del farmacéutico están muy relacionados con errores en despacho de medicamentos e irregularidades en el manejo de la receta médica. Sin embargo, los nuevos retos de una profesión que se encuentra en proceso de ampliación de sus competencias, y que tiene una responsabilidad con los sistemas de salud cada vez mayor, originan inevitablemente nuevos escenarios de responsabilidad, de la misma manera como se produce actualmente con los médicos y otros profesionales en salud (Sanchez-Caro y Abellán, 2004).

\section{Deber de cuidado}

De acuerdo con el artículo 30 del Código Penal costarricense, nadie puede ser sancionado por un hecho expresamente tipificado en la ley, si no lo ha realizado con dolo, culpa o preterintención. Para analizar la responsabilidad de una persona por la comisión de un hecho delictivo, es necesario que se analice la tipicidad, la antijuricidad y la culpabilidad de la conducta realizada por el sujeto.

En el caso de los delitos dolosos, la tipicidad está definida a partir de la comparación de la conducta realizada con la conducta descrita en la norma penal, se requiere individualizar la acción prohibida por el fin perseguido por ella. En cambio, el tipo culposo lo hace en razón de que la programación de la causalidad, por violar un deber de cuidado, produce el resultado típico. El resultado contrario de derecho puede producir el deterioro de la salud del sujeto e incluso la muerte.

Lo anterior no significa que la acción imprudente no tenga finalidad: simplemente, no individualiza la conducta prohibida en razón de esa finalidad, sino en razón de la falta de cuidado con que se produce. Para el análisis de los delitos culposos es necesario determinar la finalidad de la conducta, dado que es indispensable identificar cuál es el deber de cuidado que incumbía al agente, porque no hay un cuidado debido único para todas las acciones (Zafaroni, 2005).

Dentro de los delitos culposos se incluyen el homicidio, lesiones levísimas, leves, graves y gravísimas. La doctrina reconoce que la ubicación de la imprudencia dentro del análisis del tipo penal y el análisis del deber objetivo de cuidado fue propuesta por Karl Engisch, en su obra Untersuchungenüber Vorsatzund Fahrlässigkeitim Strafrecht, de 1930. Esta posición fue luego desarrollada por la concepción finalista del delito. En esta concepción finalista se fundamenta el artículo 279 del Código Penal costarricense, el cual hace referencia expresa al caso de los 
profesionales que actúan de manera imprudente, negligente o por inoperancia del agente, al señalar que:

Cuando alguno de los hechos (...) fuere cometido por imprudencia o negligencia o por inoperancia en el arte o profesión del agente o por inobservancia de reglamentos, se impondrán además de las penas consignadas, la de inhabilitación para el ejercicio de la profesión, oficio, arte o similares que desempeñe, de uno a cuatro años.

La imprudencia se considera un elemento del tipo y junto al desvalor del resultado se debe valorar un desvalor de la acción que está integrado por una infracción al deber de cuidado. El deber de cuidado es el modelo sobre el cual se compara la acción del sujeto y así valorar su tipicidad, es el punto de referencia sobre el cual se analiza la conducta y el resultado.

\section{Lex artis}

Con el fin de valorar la conducta profesional que produjo un resultado lesivo se han utilizado las llamadas "Reglas de cuidado" las cuales ordenan de forma general una determinada actividad. Existen reglas de cuidado que son emitidas en forma de leyes o reglamentos y que ordenan de forma general situaciones en las que puede haber un riesgo. Para el caso del despacho de medicamentos se emitió la Ley sobre estupefacientes, sustancias psicotrópicas, drogas de uso no autorizado, actividades conexas, legitimación de capitales y financiamiento al terrorismo y su reglamento, los cuales regulan el despacho de medicamentos psicotrópicos y estupefacientes. Además se promulgó el Reglamento de Establecimientos Farmacéuticos Privados el cual contiene elementos que orientan el despacho de medicamentos.

Según el criterio de la Sala Tercera de la Corte Suprema de Justicia, la lex artis no es algo que se encuentre sistematizado, e incluye también aspectos como la costumbre o experiencias.

\footnotetext{
Las pautas de la lex artis médica están compuestas tanto por el conocimiento sistematizado y validado por la ciencia médica, como también por las normas de experiencia o costumbres que, si bien no se encuentran formalmente sistematizadas mediante formulaciones teórico-científicas, gozan de un alto grado de reconocimiento, reiteración y vigencia en los distintos ámbitos y procedimientos vinculados con las ciencias de la salud y con la praxis en que estas se implementan (Resolución 2010-00950).
}

Debido a la diversidad de situaciones posibles, las normas no pueden abarcar cada unas de las actuaciones que podría realizar un profesional y que produzcan un daño. El continuo avance o cambio en la ciencia y las técnicas empleadas, 
imposibilita que se emitan normas jurídicas en la mayoría de las actividades sanitarias, lo cual impide su estandarización. Por lo anterior, existen muchos aspectos del ejercicio profesional que no están regulados jurídicamente, sino que emanan de asociaciones, colegios profesionales y organismos internacionales.

La Sala Tercera de la Corte Suprema de Justicia se ha pronunciado respecto a la relevancia de la lex artis en la resolución de los casos de culpa.

Los delitos culposos se desarrollan mediante tipos penales abiertos, cuyos alcances deben ser "rellenados" o "delimitados" mediante la actividad del juzgador. Con ese fin, según lo requiere el principio de legalidad penal, la acción o la omisión típica en esta clase de delitos debe ser delimitada claramente mediante la consideración de las denominadas reglas o pautas de la lex artis médica (...) en los delitos culposos de mala praxis médica el juez no tiene la potestad de definir la acción típica desde criterios antojadizos o subjetivos porque ello vulneraría el principio de legalidad penal (...) lo primero que debe dilucidarse mediante la fundamentación jurídica del juez penal es cuáles reglas de la lex artis regían al momento de los hechos para la labor encargada a las y los profesionales (...) En principio, una acción real de tales profesionales de la salud será típica si, y solo si, por acción u omisión, se aparta de aquellas pautas de comportamiento, objetivamente predeterminadas mediante las reglas de la lex artis médicas aplicables en aquel contexto. Al juez le corresponde delimitar, más no definir según su voluntad, aquellas acciones a las que, en virtud de las pautas médicas vigentes al momento de los hechos, están obligados los profesionales (Resolución 2010-00950).

Estas regulaciones corresponden a situaciones genéricas de la labor del profesional y normalmente se basan en el consenso de expertos en la materia. Dentro de estos se encuentran las guías y los protocolos. Tal como señala Villacampa, esta codificación escrita de reglas técnicas busca evitar la arbitrariedad de pericias individuales sobre la base de usos generalizados indemostrables (Villacampa, 2003).

Se ha criticado que estos protocolos de actuación no necesariamente son objetivos, sino que podrían existir intereses particulares en su elaboración que no necesariamente responden a un bienestar del paciente, sino que podrían responder a intereses económicos. Cabe también la posibilidad que la evidencia científica que origina los protocolos sea divergente 0 carente de rigurosidad científica, principalmente debido a los constantes cambios que se producen en el campo médico. Por estas razones, el uso de normas de cuidado se ha considerado importante para valorar la conducta, sin embargo, solamente tienen un papel indiciario en la violación al deber de cuidado. 
Para determinar el deber de cuidado, principalmente en las profesiones sanitarias, se utiliza el concepto de Lex artis para referirse a normas técnicas escritas o no escritas que generalmente son aceptadas en la disciplina que se practica. Según López Timoneda: “...La Lex Artis se refiere a una serie de reglas técnicas siguiendo los estándares aceptados y aprobados en el ejercicio de la profesión, teniendo en cuenta las circunstancias y condiciones en que hubieron de efectuarse y la situación del paciente en cada caso; en definitiva, es hacer bien las cosas o realizar una actuación profesional correcta (...)" (López, 1995).

La Sala Primera de la Corte Suprema de Justicia ha incluido el concepto de Lex Artis y ha indicado que:

Debe regirse por la "Lex Artis", que consiste en una actividad encuadrada en las pautas y reglas que natural y armoniosamente derivan del estado del conocimiento técnico-científico actual, integrando también normas de la deontología. Su invocación supone una referencia no sólo a normas de carácter profesional, sino también a criterios éticos. La obligación médica es poner todos los conocimientos a la orden del paciente a los efectos de mejorar su salud (obligación de medios) y cuando son insuficientes, deben realizarse interconsultas, trabajando interdisciplinariamente a efectos de buscar lo adecuado para su beneficio, pero no puede asegurársele, la cura total (obligación de resultados). En este sentido, rige el principio de que el paciente ingresa al centro hospitalario para curarse, no para enfermarse más (Resolución 000875).

Esta sentencia de la Sala Primera hace referencia a que la Lex Artis además de normas técnicas incluye también normas deontológicas. Sin embargo, si se busca establecer un parámetro lo más objetivo posible de valoración de una conducta debería hacerse con los criterios objetivos y basados en evidencia científica y no de acuerdo con elementos éticos que incluyen un alto grado de subjetividad.

\section{Configuración del delito}

Con el fin de determinar la violación al deber de cuidado es necesario el uso de un baremo objetivo que asegure que la acción ha superado el nivel de riesgo permitido, produciendo una lesión para el bien jurídico tutelado. Según Luzón Peña no se infringe el deber de cuidado y, por lo tanto, no se puede hablar de imprudencia, cuando el sujeto ha adoptado suficientes medidas de prudencia y control del peligro, es decir, cuando se mantiene en los límites del riesgo permitido (Luzón, 1996).

Por lo tanto, se utiliza una abstracción denominada "hombre medio ideal" del cual se desprende una conducta debida, construida a partir de la conducta que observaría un hombre diligente de ese mismo contorno o ámbito socio-laboral en esa 
misma situación y con similares conocimientos y aptitudes, del sentido común, las normas escritas y no escritas que debería cumplir un profesional actuando en el lugar del sujeto que produjo la lesión.

La infracción de una norma de cuidado no fundamenta en sí misma una falta al deber objetivo de cuidado, debe considerarse además si el sujeto es capaz de prever y poder evitar la producción del resultado y por lo tanto la conformación del tipo penal. Según propone Feijoo, se ha equiparado o sustituido, en algunos casos, la violación de la norma de cuidado por la idea de creación de un riesgo jurídicamente desaprobado, no permitido, típicamente relevante o socialmente inadecuado (Feijoo, 2001).

Por lo tanto, es necesario valorar las reglas penales, las normas de cuidado extrapenales y luego las circunstancias de tiempo y lugar acaecidas en la situación concreta. Las normas de cuidado podrían dar idea del grado de previsibilidad del resultado de una determinada conducta. Para que se produzca una infracción al deber de cuidado es necesario que haya previsibilidad objetiva de realizar el tipo a través de una acción concreta, ya que cuando exista esta posibilidad se puede exigir que se actúe con el fin de evitar ese resultado lesivo. Es necesario que concurra la previsibilidad para que se complete la parte subjetiva del tipo. Además de la parte objetiva del tipo.

\section{Despacho de medicamentos: acto profesional farmacéutico}

El despacho de medicamentos, es una actividad exclusivamente del profesional en farmacia que tiene pasos definidos por lo cual es posible darle contenido a la lex artis en el caso concreto de esta actividad profesional. El despacho de medicamentos consiste en la entrega a un paciente de un medicamento prescrito para el alivio o cura de una enfermedad, el cual deber ser realizado por un profesional y debe darse al paciente con información acerca del medicamento, forma de uso, precauciones y demás información que lleve a un uso racional del medicamento.

La ley obliga al farmacéutico a rechazar el despacho de recetas que no cumplan con los requerimientos científicos, entre ellos, la prescripción de una dosis errónea, la presencia de interacciones medicamentosas, así como las legales y reglamentarias, por ejemplo esté firmada por el médico y si es psicotrópico o estupefaciente esté prescrito en la correspondiente receta, entre otros.

El despacho de recetas de medicamentos requiere un profundo conocimiento 
de los medicamentos, su aplicación clínica y los métodos industriales de fabricación. Se compone esencialmente de cuatro pasos:

1. Lectura, interpretación y validación de la prescripción.

Los medicamentos contenidos en la receta médica deben ser analizados detalladamente por el profesional y garantizar que no existen interacciones entre los medicamentos que consume el paciente, confirmar la dosis del medicamento de acuerdo con las condiciones del paciente y la patología para la cual va a ser utilizado. Luego de asegurarse que estos aspectos estén conformes a lo que establece la evidencia científica, procede a preparar los medicamentos.

2. Preparación de los medicamentos para su entrega.

Los medicamentos deben estar almacenados de acuerdo con las condiciones necesarias para su conservación, sea que estas requieran temperaturas específicas como las vacunas o estar alejados de fuentes de luz o calor. Debe evitarse el deterioro y la entrega a un paciente de medicamentos vencidos. Puede requerirse la preparación del medicamento in situ.

3. Entrega de los medicamentos al paciente.

La entrega de los medicamentos al paciente con instrucciones y consejos específicos, sólo la puede realizar el profesional farmacéutico. Este paso es uno de los más delicados dado un error en la calidad o cantidad del medicamento, constituye un delito tipificado expresamente en el Código Penal, el artículo 273 señala que.

Será reprimido con veinte a cien días multa el que, estando autorizado para el expendio de sustancias medicinales, las suministrare en especie, calidad o cantidad no correspondiente a la receta médica o diversa de la declarada o convenida.

El Tribunal de Casación Penal en resolución de las quince horas con diez minutos del once de junio de mil novecientos noventa y ocho en resolución 000406 , consideró que este delito recae necesariamente en el profesional farmacéutico a cargo del despacho de medicamentos, indicó que la norma exige una condición típica binaria, porque no basta el suministro de la sustancia medicinal, sino, además de ello, la exigencia de estar el sujeto activo en el momento de la acción a cargo de su expendio. Por lo cual el personal de apoyo en la farmacia no podría fungir como sujeto activo de la acción que tipifica el Código Penal, dado que el único responsable del despacho es el profesional farmacéutico.

4. Seguimiento de la farmacoterapia. 
El seguimiento farmacoterapéutico se define como el servicio profesional que tiene como objetivo la detección de problemas relacionados con medicamentos (PRM), para la prevención y resolución de resultados negativos asociados a la medicación (RNM). Este servicio implica un compromiso, y debe proveerse de forma continuada, sistematizada y documentada, en colaboración con el propio paciente y con los demás profesionales del sistema de salud, con el fin de alcanzar resultados concretos que mejoren la calidad de vida del paciente (Foro de Atención Farmacéutica, 2008). Dado que es una tarea que corresponde realizar a un profesional farmacéutico y se encuentra implícito dentro del acto de despacho de medicamentos, sería posible responsabilizar penalmente al farmacéutico en el caso que el seguimiento no se lleve a cabo de manera adecuada.

\section{División del trabajo}

El desarrollo tecnológico ha influido en las ciencias médicas de forma tal que ha generado un mayor conocimiento de los procesos de los estados salud y enfermedad, esto ha producido una alta especialización de los profesionales en salud. La división que se dio hace varios siglos entre la medicina y la farmacia se debió principalmente a la especialización de cada profesional. Actualmente ambos campos han logrado un alto grado de especialización que hace más complejas las relaciones entre ambos gremios, principalmente en el despacho de los medicamentos. El ámbito de competencia de cada profesional puede dividirse en vertical y horizontal.

Las relaciones horizontales en general son las que mantienen personas del mismo grado académico y mismo nivel en el organigrama de una Institución (Rodriguez, 2012). Se incluyen las relaciones farmacéutico-farmacéutico y médicofarmacéutico que se presentan en el despacho de medicamentos. En este tipo de relaciones no existe relación jerárquica, ni deberes de obediencia en ningún sentido, se caracteriza por la autonomía de cada sujeto en su campo, en el que se aplican los conocimientos técnico-académicos y los obtenidos a través de la experiencia. Una particularidad de la relación horizontal en el despacho de medicamentos es que siempre se trabaja por un mismo paciente, en distintas fases de un mismo acto médico, diagnóstico-tratamiento, sin haber necesariamente una coincidencia en la ubicación física de los profesionales.

El otro tipo de relación que es frecuente en el ámbito médico-sanitario, que es importante en el acto del despacho de medicamentos, es la relación vertical, el caso 
de la relación farmacéutico-asistente y farmacéutico jefe-farmacéutico subordinado. Estas relaciones están definidas en el organigrama de la institución, o pueden corresponder a divisiones logísticas en los departamentos para efectos de lograr mayor orden y efectividad en el trabajo. El sujeto subordinado se encuentra obligado a obedecer a su superior en todo momento que reciba una orden de su superior (Romeo, 2007).

\section{Principio de confianza}

El principio de confianza es un elemento descrito en la doctrina que adquiere su valor en el trabajo en equipo y supone que cada miembro del equipo debe confiar en que el trabajo de su compañero será correcto, en el transcurso normal de una actividad. En general, el principio de confianza delimita el deber de cuidado, restringiendo su extensión.

La Sala Tercera de la Corte Suprema de Justicia señala que en la división de funciones de los equipos cada uno de sus miembros responde por sus propios comportamientos, que cada uno es penalmente responsable por aquellas de sus actuaciones que infringen el deber de cuidado. Sin embargo, en el caso del equipo médico indica lo siguiente:

La actuación con que uno de sus miembros falta al deber de cuidado, puede incidir en el deber de cuidado de otro. Cuando la colaboración de varias personas incide en un resultado final lesivo, no solo interesa determinar la responsabilidad individual, sino la incidencia que tiene su actuación en el deber de cuidado de otros intervinientes (Resolución 2010-00950).

El principio de confianza no es aplicable para delimitar todos los casos de responsabilidad compartida, existen situaciones que por su naturaleza anulan este principio (Feijoo, 2000). Para que un sujeto se ampare en el deber de confianza para delimitar su responsabilidad, debe comprobarse que, en ningún momento, el sujeto quebrantó su deber de cuidado y actuó en todo momento apegado a la lex artis. En segundo lugar, el principio de confianza tiene efecto en situaciones que transcurren normalmente, de tal manera que no sea posible prever que algunos de los miembros van a actuar imprudentemente (Romero, 2000). Si el sujeto es capaz de prever circunstancias anómalas con algún miembro del equipo tendrá que ampliar el cuidado hasta el punto de abarcar la conducta de los demás.

Respecto a lo anterior, La Sala Tercera de la Corte Suprema de Justicia, 
describe en principio de confianza de la siguiente manera:

De manera resumida, esto significa que quien forma parte de un equipo médico, y ha desarrollado todas sus actuaciones dentro de las técnicas y procedimientos que debe observar, no infringe el deber de cuidado, aún cuando no tome precauciones especiales respecto de las eventuales infracciones al deber de cuidado por parte de otros médicos o profesionales en la salud intervinientes en el equipo o en el trabajo compartido, salvo donde tenga razones suficientes o evidentes para dudar o tomar precauciones respecto del trabajo de otros. El principio general de confianza también funciona como un motivo de exclusión de la tipicidad por no-infracción al deber de cuidado (Resolución 2010-00950).

Como se señaló anteriormente, para configurarse el delito culposo, se debe analizar el grado en que el sujeto es capaz de prever el daño que podría causar una conducta imprudente, pues es lo que permite realizar una distinción clara del dolo (Luzón, 2012). La previsibilidad de una situación anómala, que puede generar acciones imprudentes en el tratamiento de un paciente, se exige a todos los miembros del equipo, en el tanto, la desviación de la normalidad sea evidente y cada uno tenga la posibilidad de reconocer determinadas circunstancias, partiendo de la idea de que no todos los miembros de grupo poseen las mismas posibilidades de prever situaciones que pueden generar daño, se exige lo mínimo que se le exige a cualquier profesional, independientemente de su especialización (Pérez, 2012).

Por ejemplo, la capacidad de detectar el estado de embriaguez de alguno de los miembros del equipo, es un situación que se espera que cualquier profesional sanitario tenga, independientemente de su especialidad o experiencia y es esperable también, que en una situación de este tipo, el profesional extienda su deber de cuidado hasta abarcar la conducta del otro, anulando el principio de confianza. Si el profesional deja que un sujeto que no se encuentra en uso de sus plenas facultades, realice sus funciones, estaría actuando de manera imprudente, y no podría apelar al principio de confianza en su defensa, en caso de un resultado lesivo para el paciente.

El caso contrario sucede en una interconsulta, donde un médico solicita a un farmacéutico un criterio técnico sobre el tratamiento de un paciente en particular, el médico debe confiar en el criterio de su compañero, siempre y cuando no exista una situación que evidencie su falta de competencia. Volviendo al ejemplo anterior, si el médico, se comunica con el farmacéutico y detecta un estado de embriaguez, en ese momento, el médico estaría actuando de manera imprudente al utilizar el criterio de su compañero, luego de prever que esto puede generar un daño para el paciente. 


\section{Alcance del Principio de Confianza en el despacho de medicamentos}

El despacho de medicamento puede ser analizado según la relación laboral desde la cual sea abordado el tema. Los actores en el acto de despacho de medicamentos son: el médico prescriptor, el farmacéutico y el técnico o dependiente de la farmacia. A continuación se analizará la validez del principio de confianza en las relaciones horizontales médico-farmacéutico y farmacéutico-farmacéutico, y en la relación vertical farmacéutico- técnico de farmacia.

\subsection{Relación médico-farmacéutico}

La prescripción de un medicamento es resultado de una decisión que se motiva en el conocimiento profesional la realiza, el principio de confianza debe operar respecto a la certeza del diagnóstico ya que es el prescriptor el que realiza la revisión del estado general del paciente, análisis de sintomatología y quien tiene los elementos técnicos necesarios para relacionar estos signos y síntomas con un determinado estado de salud de un paciente. Cabe destacar que a pesar de que el médico es el profesional en salud que usualmente realiza las prescripciones, la Ley General de Salud faculta a los odontólogos y las enfermeras obstetras a realizar prescripciones en su ámbito de competencia.

Como se analizó anteriormente, el farmacéutico puede desconfiar de la prescripción del médico y/o diagnóstico, en casos fuera de la normalidad, cuando sea previsible que el prescriptor actúa imprudentemente, y no solo para el farmacéutico, sino para cualquier miembro del equipo de trabajo, independientemente de su área de competencia. Por ejemplo: en la prescripción de un medicamento oncológico por parte de un médico general, es evidente que el médico general no tiene los conocimientos ni la preparación para diagnosticar una patología tan compleja ni para prescribir medicamentos de esta categoría, porque esto requiere un nivel de especialización mayor.

Sin embargo, durante el curso normal de las situaciones de despacho de medicamentos, el principio de confianza se anula respecto a la selección adecuada del medicamento, la dosis y la reacción del paciente a la terapia farmacológica, dado que es responsabilidad del farmacéutico realizar un control cruzado de la prescripción de un medicamento con la patología que presenta, es decir el farmacéutico debe validar la idoneidad de la prescripción para poder realizar su despacho en virtud del resguardo de la salud del paciente al cual se le está prescribiendo el medicamento. 
No es razonable pensar que el farmacéutico debe realizar la revisión de una prescripción, asumiendo que es correcta, esto eliminaría completamente la razón de ser del profesional en farmacia en el acto de despacho de medicamentos; incumpliendo con lo delegado por la Ley, como lo justifica el Tribunal Superior Penal de Pérez Zeledón en la sentencia número 87-1995 a las veintiuna horas y treinta minutos del cuatro de junio de mil novecientos ochenta y cinco refiriéndose a la conducta del profesional en farmacia:

La coimputada estaba en la obligación de rechazar el despacho de la receta, por cuanto la misma no era conforme a las exigencias científicas (...) no tomó las previsiones supra mencionadas a pesar de que son propias del farmacéutico (...) ya que tiene la obligación y la autoridad profesional para impedir que se despache la receta en la farmacia.

En el caso de resultar un daño para un paciente, la responsabilidad recae en ambos profesionales, a pesar de ser el profesional farmacéutico quien autoriza el despacho de un medicamento mal prescrito, debido a que quien se refugia en el principio de confianza para su defensa debe actuar con prudencia todo el tiempo. Lo anterior, también es sujeto de análisis del Tribunal en el caso supra citado, sobre lo cual indica lo siguiente:

En todo caso en la especie conforme se dirá existió coauditoría, es decir, la culpa es compartida por el hecho de que el farmacéutico no hiciera la advertencia, no exime de culpa al médico.

En este caso el Tribunal determina que el médico tratante actuó con impericia y el profesional en farmacia con negligencia, al respecto el tribunal indica lo siguiente:

Por otro lado, el artículo 56 de la Ley General de Salud dispone que los farmacéuticos está en la obligación de rechazar el despacho de toda receta que no se conforme a las exigencias científicas y reglamentarias, de modo que no se está exigiendo a la imputada una cuota excesiva de responsabilidad como se afirma en el recurso, sino una elemental prudencia y cuidado a la hora de despachar recetas.

Por lo tanto, en el acto de despacho de medicamentos, no se parte del supuesto de que el prescriptor seleccionó el medicamento correcto, en la dosis y frecuencia correcta y que el tratamiento no tiene interacciones medicamentosas con otros fármacos utilizados por el paciente, esto no permitiría una revisión técnicocientífica de la receta y eliminaría el sentido de que exista un profesional que garantice que el paciente tenga doble seguridad que va a recibir el tratamiento adecuado para el alivio de sus afecciones. Por la naturaleza del acto, se anula el 
principio de confianza, a pesar de ser una relación entre dos profesionales de igual rango.

\subsection{Relación farmacéutico-farmacéutico}

La distribución de labores entre profesionales para el despacho de una receta para un mismo paciente es poco frecuente en la práctica farmacéutica; se da en pocos casos o situaciones. Una de ellas es cuando se divide el acto de despacho y se reconocen dos partes que son responsabilidad del farmacéutico: la revisión inicial, que comprende la lectura, interpretación y validación de la prescripción, y la revisión final, que implica la entrega de los medicamentos al paciente. La otra situación es en el despacho de preparaciones magistrales.

En algunos momentos, la revisión inicial y la revisión final están a cargo de dos profesionales en farmacia distintos: el profesional que realiza revisión final corrobora que el medicamento corresponda a lo etiquetado y lo entrega al paciente correcto con las indicaciones pertinentes, y debe confiar en que la dosis y el medicamento son los correctos de acuerdo con el diagnóstico del médico, función que realizó su compañero durante la revisión inicial de la receta. En este caso se requiere que opere el principio de confianza entre los dos profesionales y se presuma que cada uno observa y actúa conforme a la lex artis, a menos que uno de los dos violente las normas técnicas de manera evidente para el otro.

En el despacho de preparaciones magistrales, generalmente participa, o es deseable que participen varios profesionales en farmacia. Un farmacéutico realiza los cálculos pertinentes para la lograr la cantidad de principio activo prescrito en la forma farmacéutica y otro farmacéutico la prepara. En este caso el farmacéutico que prepara debe confiar en que las cantidades indicadas de principio activo por su compañero son las correctas, es decir debe aplicar el principio de confianza, a menos que sea previsible que su compañero no actúa de manera prudente, apegado a las normas de cuidado establecidas.

8.3 Relación farmacéutico- personal de apoyo

La colaboración de personal de apoyo en el despacho de medicamentos es indispensable en algunos servicios de farmacia con gran afluencia de pacientes. El acto de despacho como tal debe ser realizado por el farmacéutico, sin embargo, algunas de las operaciones durante la preparación de los medicamentos para su despacho puede ser realizado por personal de apoyo, bajo las condiciones que indica el artículo 48 de la Ley General de Salud: 
Art. 48. Los profesionales en Ciencias de la Salud, a que se refiere el artículo 40, sólo podrán delegar, o asociarse para delegar algunas de sus funciones a personas debidamente capacitadas, lo cual harán en todo caso bajo su responsabilidad, y de conforme a lo reglamentado de esta ley y del respectivo Colegio.

Es claro que la ley contempla el delegar funciones bajo un criterio profesional, sin embargo, también es clara en que no exime de responsabilidad a los profesionales por errores que pueda cometer el personal de apoyo. El personal de apoyo no tiene los conocimientos ni la preparación para reconocer un error en el proceso de despacho, por lo que es una situación donde no opera el principio de confianza. El farmacéutico debe revisar todas las operaciones que realizan los colaboradores, de lo contrario, estaría infringiendo su deber de cuidado.

\section{Conclusiones}

La responsabilidad profesional en farmacia en el acto de despacho de medicamentos tiene fundamento en una violación al deber de cuidado, el cual se analiza a partir de la lex artis. Cada etapa del acto de despacho requiere un análisis diferente, debe considerarse que es un acto complejo en el que siempre participan varios sujetos, por lo cual el principio de confianza varía dependiendo de la etapa. En la etapa de validación de la prescripción, el principio de confianza se rompe respecto al profesional que prescribe y el farmacéutico debe desconfiar siempre de la prescripción. Asimismo, si participa personal de apoyo, el principio de confianza no opera. En la segunda etapa, en el que participan varios profesionales en farmacia, se debe partir del principio de confianza.

\section{Referencias}

BERNARDO FEIJÓO, Sánchez. El principio de confianza como criterio normativo de imputación en el derecho penal: Fundamento y consecuencias dogmáticas. Revista de Derecho Penal y Criminología, (Extra n-1): 93-138, 2000. ISSN: 0121-0483

COSTA RICA. Código Penal. La Gaceta 257, del 15 de noviembre de 1970. Alcance $120 \mathrm{~A}$.

COSTA RICA. Corte Suprema de Justicia. Sala Tercera. Resolución 2010-00950. San José, 9 de setiembre del 2010. [on line] Disponible en Sistema Costarricense de Información Jurídica (SCIJ): http://jurisprudencia.poderjudicial.go.cr/SCIJ_PJ/busqueda/jurisprudencia/jur_Documento.aspx?param1=Ficha Sentencia\&nValor $1=1 \&$ nValor2 $=500768 \&$ strTipM $=\bar{T} \&$ strDirSel=directo Fecha de consulta: 15 oct. 2014. 
COSTA RICA. Corte Suprema de Justicia. Sala Primera. Resolución 000875. San José, 14 de diciembre de 2007. [on line] Disponible en Sistema Costarricense de Información Jurídica (SCIJ): http://jurisprudencia.poderjudicial.go.cr/SCIJ PJ/busqueda/jurisprudencia/jur Documento.aspx?param1=Ficha

Sentencia\&nValor1=1\&nValor2=400897\&strTipM=T\&strDirSel=directo $\quad$ Fecha de consulta: 15 oct. 2014.

COSTA RICA. Ley General de Salud. 1973. La Gaceta 222, del 24 de noviembre de 1973. Alcance 172. [on line] Disponible en Sistema Costarricense de Información Jurídica

(SCIJ):

http://196.40.56.11/scij/Busqueda/Normativa/Normas/nrm texto completo.aspx?para m1=NRTC\&nValor1=1\&nValor2=6581\&nValor3=96425\&strTipM=TC Fecha de consulta: 15 oct. 2014.

COSTA RICA. Tribunal de Casación Penal de San José. Resolución 000406. San José, 11 de junio de 1998. [on line] Disponible en Sistema Costarricense de Información Jurídica (SCIJ): http://jurisprudencia.poderjudicial.go.cr/SCIJ PJ/busqueda/jurisprudencia/jur Documento.aspx?param1=Ficha

Sentencia\&nValor1=1\&nValor2=148278\&strTipM=T\&strDirSel=directo $\quad$ Fecha de consulta: 15 oct. 2014.

COSTA RICA. Tribunal Superior Penal de Pérez Zeledón. Resolución 000087. Homicidio culposo, Arroyo Valverde/ Zamora Gutiérrez y otra. San José, 4 de junio de 1985.

FEIJOO SÁNCHEZ, Bernardo. Resultado lesivo e imprudencia (1 ed). Barcelona: J.M. Bosch Editor, S.A., 2001. p. 243. ISBN13:9788476986233.

MINISTERIO DE SANIDAD, SERVICIOS SOCIALES E IGUALDAD. Consenso sobre Atención Farmacéutica. ESPAÑA. [on line] Disponible en https://www.msssi.gob.es/profesionales/farmacia/consenso/consenso.htm\#indice

Fecha de consulta: 15 oct. 2014.

GRACIA GUILLÉN, Diego. LÓPEZ TIMONEDA, Francisco. JORGE BARREIRO, Alberto. Jurisprudencia y Lex artis en Responsabilidad del personal sanitario, Cuadernos de Derecho Judicial, Monografías 1. Consejo General del Poder Judicial, Madrid: 51-134, 1995.

HORWITZ CAMPOSA, Nina. EI sentido social del profesionalismo médico. Revista Médica de Chile, 134: 520-524, 2006. ISSN 0034-9887

LUZÓN PEÑA, Diego-Manuel. Curso de Derecho Penal. Parte General. Madrid: Universitas, 1996. p. 637. ISBN 9788479910419.

LUZÓN PEÑA, Diego-Manuel. Lecciones de Derecho Penal. Parte General. Madrid: Tirant lo Blanch. 2012. p 316. ISBN ISBN13:9788490332696.

PÉREZ DEL VALLE, Carlos. La imprudencia en el Derecho Penal. El tipo subjetivo del delito imprudente. Barcelona: Atelier. 2012. p. 58. ISBN: 9788492788842.

PORTERO LAZCANO, Guillermo. Responsabilidad penal culposa del médico: fundamentos para el establecimiento de la negligencia o impericia. Revista Latinoamericana de Derecho Médico y Medicina Legal, 6 (2): 89-96, 2001. ISSN 1409-0945

RODRÍGUEZ VÁZQUEZ, Virgilio. Responsabilidad penal en el ejercicio de actividades médico-sanitarias. (1 ed). Madrid: Marcial Pons, Ediciones Jurídicas y 
Sociales. 2012. p.259-287. ISBN: 9788497688925.

ROMEO CASABONA, Carlos María. Evolución del tratamiento jurídico-penal de la imprudencia del personal médico-sanitario. Revista Jurídica de Castilla y León., 13: 243, 2007. ISSN: 2254-3805

ROMERO FLORES, Beatriz. La imputación objetiva en los delitos imprudentes. Anales de Derecho, 19: 259-278, 2001. ISSN: 1989-5992

SANCHEZ-CARO, Javier; ABELLAN, Fernando. Atención farmacéutica y responsabilidad profesional. Granada: Comares, 2004. p. 4-9. ISBN 9788484448914.

VILLACAMPA ESTIARTE, Carolina. Responsabilidad penal del personal sanitario. Pamplona: Editorial Aranzadi, S.A. 2003. p. 108. ISBN: 9788497672948.

ZAFFARONI, Eugenio Raúl, Manual de Derecho Penal: Parte General. (1 ed) Buenos Aires: Ediar, 2005. p. 423-424. ISBN: 950-574-75-8. 